\title{
Importance of Routine Histopathological Examination of Gallbladder Specimen in Detecting Incidental Malignancies
}

\author{
Archana Tiwari, ${ }^{\mathrm{a}, \mathrm{c}}$ Ramji Rai, ,,c Surendra Kumar Jain ${ }^{\mathrm{b}, \mathrm{c}}$
}

\begin{abstract}
:
Introduction: Gallbladder carcinoma is the most common cancer of biliary tree and the $5^{\text {th }}$ most common gastrointestinal malignancy. An early diagnosis is essential as this malignancy progresses silently with a late diagnosis and poor prognosis. Epidemiological studies have identified striking geographic and ethnic variation with high occurrence in Southeast Asia, yet low elsewhere in the world. Gallbladder carcinoma, in 15-30\% of patients, show no preoperative or intraoperative evidence and are detected only on histopathological examination. They are called as incidental gallbladder cancer (IGBC). The objective of this study was to find out the occurrence of IGBC in cholecystectomy specimens received in our histopathology laboratory and to analyze their clinico-pathological features. Methods: This was a prospective study carried out in the Department of Histopathology, Lumbini Medical College Teaching Hospital during a period of two years from May 2014 to April 2016. The study included 800 cases of cholecystectomized Gall bladder specimens. Result: Ninety seven percent of the specimens $(n=776)$ revealed benign pathology. Malignancy was detected incidentally in 8 cases (1.25\%). The mean age of patients with incidental Gall bladder carcinoma was 69 years $(S D=4.1)$ and $\mathrm{F}: \mathrm{M}$ ratio was 9:1. Out of 10 incidental malignancies, cholelithiasis was found in $8(80 \%)$ cases. Fundus was the most common location $(n=5,50 \%)$ and focal fragile necrotic area was most common $(n=3,30 \%)$ gross morphology. On pathological staging, all the incidentally detected malignancies $(n=10)$ were found to be in surgically resectable stages. Conclusion: IGBC was found in $1.25 \%$ of the gallbladder specimen. Detailed gross and histopathological examination of gallbladder specimen is mandatory for every cholecystectomy specimen, even for benign diseases, to detect incidental carcinoma at potentially curable stage.
\end{abstract}

Keywords: carcinoma $\bullet$ cholecystectomy $\bullet$ gallbladder $\bullet$ incidental

\section{INTRODUCTION:}

Gallbladder cancer is the most common cancer of biliary tree and the fifth most common gastrointestinal cancer. ${ }^{1}$ It is well known for its poor prognosis. The signs and symptoms of gallbladder

a - Lecturer,

b - Professor

c - Department of Pathology,

Lumbini Medical College Teaching Hospital, Palpa, Nepal

Corresponding Author:

Dr. Archana Tiwari

e-mail: archana445@gmail.com

How to cite this article:

Tiwari A, Rai R, Jain SK. Importance of routine histopathological examination of gallbladder specimen in detecting incidental malignancies. Journal of Lumbini Medical College. 2016;4(1):15-9. doi: 10.22502/jlmc.v4i1.76. carcinoma are not specific and often present late. ${ }^{2}$ Diagnosis is, therefore, often made in the advanced stage with a poor prognosis; an overall mean survival of six months and a five-year survival rate of less than 5\%. Most gallbladder carcinoma cases are suspected preoperatively or intraoperatively. Fifteen to thirty percent of patients with gallbladder carcinoma show no preoperative or intraoperative evidence of gallbladder cancer. ${ }^{3}$ These kind of carcinomas are called as incidental carcinoma. Incidental gallbladder cancer (IGBC) may be defined as a malignancy detected only on histopathological examination without prior preoperative or intraoperative suspicion of malignancy. ${ }^{4,5}$

With the introduction of laparoscopic surgery and the higher acceptance of this 
technique, gallbladders are now removed much frequently than they used to be. With the increase of cholecystectomies, the diagnosis of incidental gallbladder carcinoma has become more frequent by histopathological examination. ${ }^{5}$ In Nepal, histopathological examination of cholecystectomy specimens has become a routine practice. ${ }^{6-9}$ Gallstone disease, porcelain gallbladder, and sclerosing cholangitis are the best known risk factors for gallbladder cancer apart from some nonmodifiable factors such as increasing age, ethnicity, female gender and family history or genetics. ${ }^{9,10}$ Cholecystectomies performed with preoperative diagnosis of gallstone disease rarely results in a diagnosis of unexpected gallbladder cancer. However, at times, it is surprising to find occult malignancy among gallbladder specimens that are submitted with clinico-radiological diagnosis of benign diseases.

Surgical removal of gall bladder is common and cholecystectomized gallbladder specimens are one of the frequently received specimens in the histopathology department. ${ }^{11,12}$ However, not much is known about the rate of incidental carcinoma in such specimen in our population. Objectives of this study were: 1) to find the proportion of incidental gall bladder carcinoma in cholecystectomy specimen and 2) analyze the different pathological entities of postoperative gall bladder specimen detected during routine histopathological examination.

\section{METHODS:}

This was a prospective study, carried out at Department of Pathology, Lumbini Medical College Teaching Hospital from May 2014 to January 2016. All Gall bladder specimens submitted to the Department of Surgical Pathology for histopathological examination were included in the study.

Routine Gall bladder specimen with preoperative or intraoperative suspicion of malignancy and/or existence of gallbladder polyps detected during preoperative evaluation were categorized separately. The specimens were fixed in $10 \%$ formalin and were sectioned serially from the neck to the fundus. Routine processing of tissue sections with hematoxylin and eosin staining was done and were reviewed by authors. Tumor staging was based on the $7^{\text {th }}$ edition of the American Joint Committee on Cancer (AJCC) manual. ${ }^{13}$

Data about patients' demographic profile, preoperative and intraoperative findings, operative procedures performed, and histopathology findings were collected in Microsoft Excel 2007. Data were analyzed with SPSS 17.0 for windows. Descriptive statistics like frequency, percentage, mean, standard deviation were calculated.

\section{RESULTS:}

A total of 800 gall bladder specimens following open and laparoscopic surgeries were received during study period. Of those 800 patients, $640(80 \%)$ were women and $160(20 \%)$ were men with $\mathrm{M}: \mathrm{F}$ ratio of $1: 4$. Mean age was 45 years $(S D=13.7$, range: $9-82$ years).

Ninety seven percent $(n=776)$ of the specimens revealed benign pathology. They were chronic calculus and acalculous cholecystitis with mild to severe non specific inflammation as well as with specific changes like eosinophilic cholecystitis, follicular cholecystitis, xanthogranulomatous cholecystitis, cholesterolosis, spongioid mucosal hyperplasia, adenomatous hyperplasia, pyloric metaplasia, and intestinal metaplasia. Two cases of tubular adenoma were also detected in gallbladder submitted with surgical diagnosis of gallbladder polyp. Eight cases of calcified/porcelain gallbladder were diagnosed; all needed decalcification before processing.

Three percent $(n=24)$ of the specimen were diagnosed as gallbladder carcinoma on histopathological evaluation. Twenty patients were female and 4 were male (M:F =1:5). Out of 24 cases, malignancy was detected on histopathological evaluation incidentally in ten cases, which comprises $1.25 \%$ of total cases. These cases were not suspected of malignancy clinically on preoperative evaluation and intraoperative evaluation. Among them nine patients were female and one was male $(\mathrm{M}: \mathrm{F}=1: 9)$. Their mean age was 69 years $(S D=4.1)$. Association with cholelithiasis was observed in $8 / 10$ cases $(80 \%)$ (Table 1).

Out of ten incidental carcinomas, on gross examination, diffuse thickening of the gallbladder wall was seen in two cases and localized growth in the form of focal thickening in two cases, focal fragile necrotic area was found in three cases and mild mucosal irregularity in one case. Two gallbladder specimens were filled with necro-hemorrhagic debris and had focal fragile elevated growth. Fundus was the most common location $(n=5)$ followed by body $(n=2)$. Three cases had multi-centric tumors 
involving fundus, body, and neck of gallbladder. One case with focal thickening of gallbladder wall had in situ carcinoma and needed extensive re-sampling for confirmation of malignancy (Table 1). Histological variants of incidental carcinoma is presented in Table 2.

On pathological staging, all of the incidentally detected cases were found to be in surgically resectable stages; T2 $(n=7,70 \%)$, T1b ( $n=2,20 \%)$, and T1a $(n=1,10 \%)$. Perineural invasion was seen in five cases (Fig 1). Mucosal lesions thought to be premalignant (pyloric metaplasia and dysplasia) were detected in six cases.

\section{DISCUSSION:}

Gall bladder is one of the commonest surgical specimen received in histopathology department and most common indications for cholecystectomies are

Table 1: Clinicopathological Features of Incidental gallbladder Carcinoma.

\begin{tabular}{lc}
\hline Variables & value \\
\hline $\begin{array}{l}\text { Over all proportion of Gall bladder } \\
\text { carcinoma } n(\%)\end{array}$ & $24 / 800$ \\
M:F ratio & $13 \%)$ \\
\hline Incidental carcinomas $n(\%)$ & $10 / 800$ \\
\hline M:F ratio & $(1.25 \%)$ \\
\hline Age in year (M, SD) & $1: 9$ \\
\hline Associated with gall stones $n(\%)$ & $69(S D=4.1)$ \\
\hline Localized growth on gross examination & $8(80 \%)$ \\
\hline Diffuse thickening of Gall bladder wall & $2(20 \%)$ \\
\hline Focal fragile necrotic area & $2(20 \%)$ \\
\hline Lumen filled with necrotic debris with & $3(30 \%)$ \\
mucosal irregularity & $2(20 \%)$ \\
\hline Mild mucosal irregularity & $1(10 \%)$ \\
\hline Fundic site & $5(50 \%)$ \\
\hline Multi-centric tumors & $3(30 \%)$ \\
\hline $\begin{array}{l}\text { Association of malignancy with } \\
\text { porcelain Gall bladder }\end{array}$ & 0 \\
Precancerous lesion & $6(60 \%)$ \\
\hline Peri-neural invasion & $5(50 \%)$ \\
\hline
\end{tabular}

Table 2: Histological variants of incidental carcinoma $(N=10)$

\begin{tabular}{cc}
\hline Histological type of carcinoma & $\boldsymbol{n}(\%)$ \\
\hline Adenocarcinoma, NOS type & $6(60 \%)$ \\
Papillary adenocarcinoma & $2(20 \%)$ \\
Mucin secreting adenocarcinoma & $1(10 \%)$ \\
Poorly differentiated carcinoma & $1(10 \%)$ \\
\hline
\end{tabular}

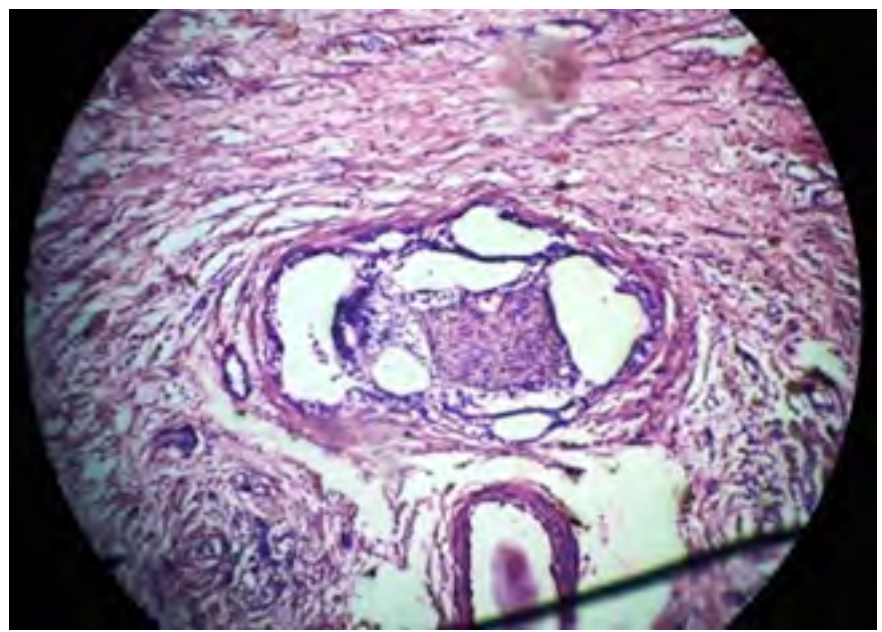

Fig 1: $H$ and $E$ stained sections of Perineural invasion in adenocarcinoma (color picture available online)

benign gallbladder diseases, such as symptomatic gallstone diseases and its complications (e.g. biliary colic, acute or chronic cholecystitis, gallstone pancreatitis, choledocholithiasis or gallbladder polyps). ${ }^{14}$ Though diagnosis of benign disease is usually made preoperatively or intraoperatively, all specimens are routinely sent for histopathological examination to know the histopathological diagnosis and exclude malignancy even though the possibility of its incidental occurrence is rare. ${ }^{4,5}$ Early detection of carcinoma of GB can save life due to its good prognosis when treated in early stage.

The incidence of gall bladder cancer varies by geographic region and racial ethnic group. The highest gallbladder cancer incidence rates worldwide have been reported in women from India, Chile, Pakistan, and Japan and in American Indians. ${ }^{15}$ Therefore, Nepal is an appropriate place to study for incidence of gallbladder carcinoma being a south Asian country and as it shares geographical, environmental and cultural similarities with those countries. Shukla et al. have shown that the high incidence of carcinoma gall bladder along the gangetic belt of northern India which is probably due to the presence of heavy metals like cadmium, mercury and lead in gall bladder specimens of patients. ${ }^{16}$

The incidence of incidentally diagnosed gallbladder cancer has been reported to vary from $0.35 \%$ up to $2.85 \%$ in different studies worldwide. ${ }^{4-8}$ In recent years, the incidence of incidental diagnosis has increased, probably because of an increase in the number of elective cholecystectomies for benign diseases in the present era of laparoscopic cholecystectomy. ${ }^{17,18}$ In the present study, the rate of 
Table 3 : Comparison of occurrence of incidental gallbladder carcinoma with different studies.

\begin{tabular}{|cccc}
\hline Study & Year & $\boldsymbol{N}$ & $\%$ \\
\hline Weinstein et al. & 2002 & 1697 & $0.35 \%$ \\
\hline Khan et al. & 2007 & 428 & $1.86 \%$ \\
\hline Deguara et al. & 2009 & 2577 & $1.0 \%$ \\
\hline Shrestha et al. & 2010 & 668 & $1.4 \%$ \\
\hline Ghimire et al. & 2011 & 783 & $1.28 \%$ \\
\hline Kalita D et al. & 2013 & 4115 & $0.44 \%$ \\
\hline Yi X et al. & 2013 & 14073 & $0.18 \%$ \\
\hline Sharma et al. & 2014 & 863 & $1.9 \%$ \\
\hline Present study & 2016 & 800 & $1.25 \%$ \\
\hline
\end{tabular}

incidental gallbladder cancer was found to be $1.25 \%$ of total cholecystectomized specimen and $41.66 \%$ of total cases of gallbladder carcinoma. Similar incidence is observed in different other studies (Table3). Deguara et al. had also reported one percent cases of incidental gallbladder carcinoma in their study of 2577 cholecystectomies. ${ }^{19}$ Khan et al. reported $1.86 \%$ of incidental gallbladder carcinomas, whereas Weinstein et al. reported $0.35 \% .{ }^{20,21}$ The rate of incidental primary carcinoma of gallbladder was $1.4 \%$ in the study of 668 cases of cholecystectomies specimens in the study of Shrestha et al. and $1.28 \%$ in the study of 783 cases by Ghimire et al., which is similar to our study. ${ }^{6-7}$ Sharma et al. found $1.9 \%$ of incidental carcinoma in cholecystectomy specimen. ${ }^{22}$

In the present study incidental carcinoma of gallbladder showed a female preponderance $(\mathrm{M}: \mathrm{F}=1: 9)$. Majority of patients in our analysis with incidental carcinoma of the gall bladder were seen in the age group of 55-85 years and mean age was 69 years. Similar results were observed in other studies from India (Pandey et al., 2001; Kapoor et al., 2003), Nepal and other Asian countries. ${ }^{6-8,23,24}$

An association of gallstones has been found in $75 \%$ to $98 \%$ of cases of incidental gallbladder carcinoma in other studies. ${ }^{8-10}$ In present study $80 \%$ $(n=10)$ of patients with incidental carcinoma had coexisting cholelithiasis.

This study showed a very high proportion of incidental gall bladder carcinoma in a relatively small sample size, which underscores the high risk of gallbladder in our population. Most of the incidentally detected carcinoma are in surgically resectable stage, with a good survival rate (Mitrovik et al. 2010; Mazer et al. 2012; Yi et al., 2013). ${ }^{25-27}$

This study showed incidental carcinomas in pathological T1a, T1b and T2 stage. Though simple cholecystectomy is said to be sufficient in stages Tis (Intra-mucosal carcinoma or carcinoma in situ.) and T1a carcinomas (Tumor grown into lamina propria), radical re-resection is strongly recommended for patients with and onwards pT1b stage (Tumor has grown into the muscularis propria). ${ }^{13,27}$ The reoperation should be performed as soon as possible, preferably within 10 days after the initial operation. ${ }^{27}$ This reflects the importance of histopathological study of all cholecystectomy specimens, irrespective of clinical impression and pathologists can provide useful information to stage the tumor and help to determine the best form of therapy.

Histopathological examination of the tumors revealed morphological findings similar to other studies. ${ }^{6-9,11,12,22,23}$ Adenocarcinoma, not otherwise specified (with mild and moderate differentiation), was found to be most common histological type (Fig 2). Porcelain gallbladder (Calcification of gallbladder) is said to be associated with10-25\% cases of carcinoma. ${ }^{28}$ In our case 5 out of 24 cases of gallbladder malignancy were associated with porcelain gall bladder. However, we could not find this association in incidental cases of malignancies. Stephen et al. reported that a calcified gallbladder is associated with an increased risk of gallbladder cancer, but at a much lower rate than previously estimated. ${ }^{28}$

\section{CONCLUSION:}

Gallbladder carcinoma is well known for its poor prognosis when diagnosed in advanced stage.

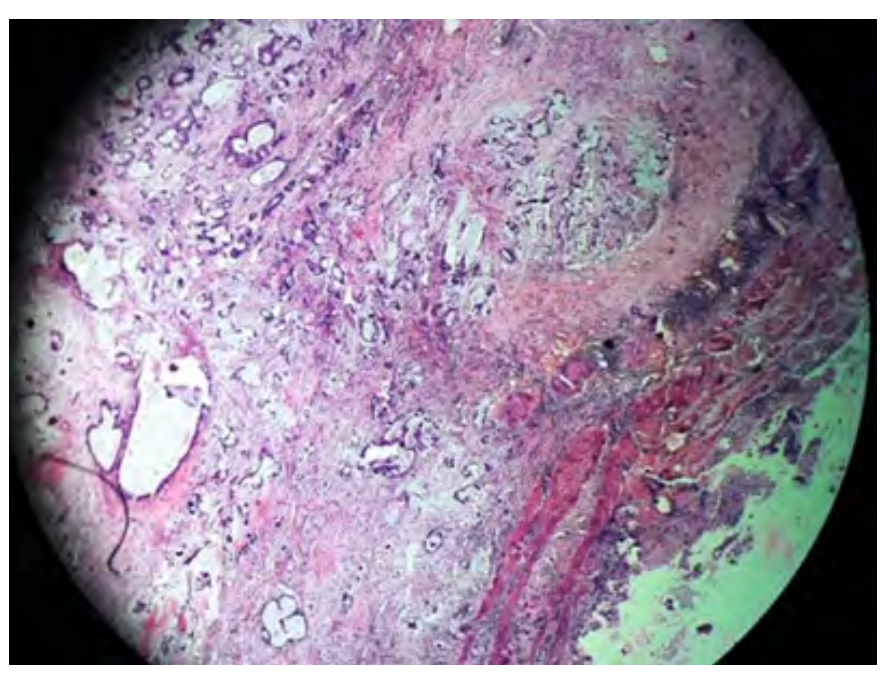

Fig 2: $H$ and $E$ stained sections from adenocarcinoma, Not Otherwise specified, $4 X$. (color picture available online) 
Importance of routine histopathological evaluation of gallbladder specimens even in benign conditions of gallbladder is well justified and supported by this study as this study has detected incidental gallbladder carcinoma in $1.25 \%$ cases. Though this value of incidental carcinoma is low, early detection of malignancy at potentially curable stage can be life saving as early diagnosis and pathological staging can guide best and appropriate management. Emphasis should be given to careful, detailed and thorough sampling of all gallbladder specimens with high degree of suspicion to detect focal neoplastic changes irrespective of clinical impression.

\section{REFERENCES:}

1. Rakic M, Patrlj L, Kopljar M, Kliček R, Kolovrat M, Loncar B, et al. Gallbladder cancer. Hepatobiliary Surg Nutr. 2014 Oct;3(5):221-6.

2. Ake Andren-Sandberg. Diagnosis and Management of Gallbladder Cancer . N Am J Med Sci. 2012 Jul;4(7):293-9.

3. Smith GCS, Parks RW, Madhavan KK, and Garden OJ. A 10-year experience in the management of gallbladder cancer. HPB. 2003;5(3):159-66.

4. Ashwin R, Cherukuri SD, Sathyanesan J, Palaniappan R, and Govindan M. Incidental gall bladder cancers: Are they truly incidental? World J Gastrointest Oncol. 2014 Dec 15;6(12): 441-3.

5. Isambert $\mathrm{M}$, Leux $\mathrm{C}$, Métairie $\mathrm{S}$, Paineau J. Incidentallydiscovered gallbladder cancer: When, why and which reoperation? J Visc Surg. 2011;148:e77-e84.

6. Shrestha R, Tiwari M, Ranabhat SK, Aryal G, Rauniyar SK and Shrestha HG. Incidental gallbladder carcinoma: value of routine histological examination of cholecystectomy specimens. Nepal Med Coll J. 2010;12(2):90-4.

7. Ghimire P, Yogi N, Shrestha BB. Incidence of incidental carcinoma gall bladder in cases of routine cholecystectomy. Kathmandu Univ Med J. 2011;34(2):3-6.

8. Baidya R, Sigdel B, Baidya NL. Histopathological changes in gallbladder mucosa associated with cholelithiasis. Journal of Pathology of Nepal. 2012;2(3):224-5.

9. Jayasundara JASB, de Silva WMM. Histological assessment of cholecystectomy specimens performed for symptomatic cholelithiasis: routine or selective? Ann R Coll Surg Engl. 2013 Jul;95(5):317-22.

10. Stinton LM, Shaffer EA. Epidemiology of Gallbladder Disease: Cholelithiasis and Cancer. Gut Liver. 2012 Apr;6(2):172-87.

11. Matthyssens LE, Ziol M, Barrat C, Champault GG. Routine surgical pathology in general surgery. Br J Surg. 2006;93(3):362-8.

12. Lohsiriwat V, Vongjirad A, Lohsiriwat D. Value of routine histopathologic examination of three common surgical specimens: appendix, gall bladder, and hemorrhoid. World J Surg. 2009;33(10):2189-93.

13. Edge SB, Byrd DR, Compton CC, Fritz AG, Greene FL, Trotti A, eds. AJCC Cancer Staging Manual. New York: Springer; 2010.

14. Potts JR 3rd. What are the indications for cholecystectomy? Cleve Clin J Med. 1990 Jan-Feb;57(1):40-7.
15. Hundal R, Shaffer EA. Gallbladder cancer: epidemiology and outcome. Clin Epidemiol. 2014;6:99-109.

16. Shukla VK, Prakash A, Tripathi BD, Reddy DCS, Singh S. Biliary heavy metal concentrations in carcinoma of the gall bladder: case-control study. Br Med J. 1998;317:1288-9.

17. Panebianco A, Volpi A, Lozito C, Prestera A, Lalongo $\mathrm{P}$, Palasciano N. Incidental gallbladder carcinoma: our experience. G Chir. 2013 May-Jun;34(5-6):167-9.

18. Varsheney S, Buttirini G, Gupta R. Incidental carcinoma of the gallbladder. EJSO. 2002;28:4-10.

19. Deguara J, Borg CM, Laferia G. Incidental gallbladder carcinoma in the Maltese Archipelago. Malta Med J. 2003;15(Suppl 1):1728-9.

20. Khan MA, Khan RA, Siddiqui S, Maheshwari V. Occult Carcinoma of Gallbladder: incidence and role of simple cholecystectomy. JK Pract. 2007;14:22-3.

21. Weinstein D. Incidental Finding of Gallbladder Carcinoma. Isr Med Assoc J. 2002;4(5):334-6.

22. Sharma JD, Kalita I, Das T, Goswami P, Krishnatreya M. A retrospective study of post-operative gall bladder pathology with special reference to incidental carcinoma of the gall bladder. Int J Res Med Sci. 2014;2(3):1050-3.

23. Pandey M, Pathak AK, Gautam A, Aryya NC, Shukla VK. Carcinoma of the gallbladder: a retrospective review of 99 cases. Digest Dis and Sci. 2001;46:1145-51.

24. Kapoor VK, McMichael AJ. Gallbladder cancer: an 'Indian' disease. Natl Med J Ind. 2003;16(4):209-13.

25. 28. Mitrović F, Krdzalić G, Musanović N, Osmić H. Incidental gallbladder carcinoma in regional clinical centre. Acta Chir Iugosl. 2010;57:95-7.

26. Mazer LM, Losada HF, Chaudhry RM, Donohue JH, Kooby DA, Nagorney DM, et al. Tumor characteristics and survival analysis of incidental versus suspected gallbladder carcinoma. J Gastrointest Surg. 2012;16(7):1311-7.

27. Yi X, Long X, Zai H, Xiao D, Li W, Li Y. Unsuspected gallbladder carcinoma discovered during or after cholecystectomy: focus on appropriate radical reresection according to the T-stage. Clin Transl Oncol. 2013;15(8):652-8.

28. Stephen AE, Berger DL. Carcinoma in the porcelain gallbladder: a relationship revisited. Surgery. 2001;129(6):699-703. 